\title{
Le cycle de développement de Renicola lari J. Timon-David, 1933 (Trematoda, Renicolidae)
}

\author{
par G. PREVOT * et P. BARTOLI * \\ * Département de Zoologie et Ecologie, Faculté des Sciences et Techniques St-Jérôme, \\ Rue Henri-Poincaré, F 13397 Marseille Cedex 4.
}

\section{Résumé.}

Le cycle biologique de Renicola lari J. Timon-David, 1933, est décrit et illustré. Les sporocystes se développent dans la masse viscérale de Cerithium mediterraneum et $C$. rupestre (Mollusca Prosobranchia). Les cercaires planctoniques sont avalées par le deuxième hôte intermédiaire Atherina boyeri ou A. hepsetus (Poissons Téléostéens Mugiliformes); elles perforent la paroi intestinale du Poisson puis s'enkystent en métacercaires dans le foie. L'adulte a été retrouvé dans les reins de Larus argentatus et $L$. ridibundus indemnes contaminés expérimentalement avec des métacercaires naturelles.

Les cercaires de Renicola lari s'éloignent des cercaires du type Plagiorchide par l'absence de stylet, la grande taille de la vessie excrétrice et leur développement en métacercaires chez les Poissons. Elles se rapprochent des cercaires du type Rhodométope par la forme et les dimensions de la vessie excrétrice mais s'en distinguent par l'absence de voiles sur la queue.

\section{Summary.}

\section{Life-cycle of Renicola lari J. Timon-David, 1933 (Trematoda, Renicolidae).}

This paper deals with life-cycle of Renicola lari J. Timon-David, 1933. Description and illustration are given. Sporocyst development occurs inside the visceral mass of Cerithium mediterraneum and C. Rupestre (Mollusca Prosobranchia). Cercariae are planktonic; they are swallowed up by the second intermediate host, Atherina hepsetus and A. boyeri (Teleostean fishes). Cercariae perforate intestine wall. Metacercariae are encysted in liver. Adult flukes have been recovered in kidneys of uninfected Larus argentatus and L. ridibundus after experimental contamination with naturally infected fishes.

Cercariae of Renicola lari differ from Plagiorchid type cercariae by the following characters: stylet lacking; very large excretory bladder; they develop into metacercariae in fishes. Cercariae of $R$. lari seem to Rhodometope type cercariae; they have the same excretory bladder. Cercariae of $R$. lari differ from Rhodometope cercariae in lacking fins on their tail.

Accepté le 5 juillet 1978. 
L'inventaire faunistique des Trématodes Digénétiques de la lagune du Brusc (Var), actuellement en cours de réalisation, nous a amené à découvrir une cercaire de Renicolidae. Le développement de cette cercaire en métacercaire a été réalisé expérimentalement chez des Poissons Athérinidés. Les métacercaires naturelles ont été trouvées dans le foie de ces mêmes Poissons.

Le lien entre la métacercaire et le Trématode adulte a été établi grâce à la contamination d'hôtes définitifs indemnes avec des métacercaires de Poissons infestés naturellement et capturés dans la lagune du Brusc.

Nous nous proposons ici de décrire le parasite à différentes étapes de son développement.

\section{Sporocyste}

\section{Hôtes :}

Cerithium mediterraneum Deshayes et C. rupestre Risso; Mollusques Prosobranches Cerithidae.

\section{Habitat :}

Les sporocystes occupent la majeure partie de la masse viscérale, c'est-à-dire la glande digestive et la gonade.

\section{Prévalence :}

Elle varie avec les saisons. Le pourcentage maximum d'individus contaminés s'observe chez Cerithium mediterraneum; de $1 \%$ en juillet, il s'élève à $2 \%$ en août, décroît jusqu'à $0,5 \%$ en septembre et s'annule en octobre. Des fluctuations semblables de la prévalence sont observées chez $C$. rupestre mais le taux est moins important: $0.5 \%$ en juillet, $1 \%$ en août, $0,5 \%$ en septembre. Nous avons observé un cas de double infestation chez un $C$. mediterraneum avec le Microphallidae, Maritrema misenensis (A. Palombi, 1940).

\section{Description :}

Le sporocyste primaire n'a pas été observé.

Les sporocystes secondaires (fig. 1) ont une couleur jaune. De forme cylindrique, ils sont arrondis aux extrémités et coudés de différentes façons. L'orifice de ponte est situé à l'une des deux extrémités.

Dimensions (*): $520-1460(1000) \times 130-280(225)$.

(*) Les dimensions sont exprimées en microns $(\mu \mathrm{m})$. Le premier chiffre indique la valeur minimale, le second la plus grande longueur; la taille moyenne figure entre parenthèses. 
Les sporocystes sont emballés dans un tissu fibreux qui les maintient très fortement adhérents les uns aux autres. Leur paroi est nettement composite et d'épaisseur importante au niveau de l'orifice de ponte (environ $20 \mu \mathrm{m}$ ). Ces sporocystes renferment de cinq à trente cercaires, la moyenne se situant aux environs de quinze.

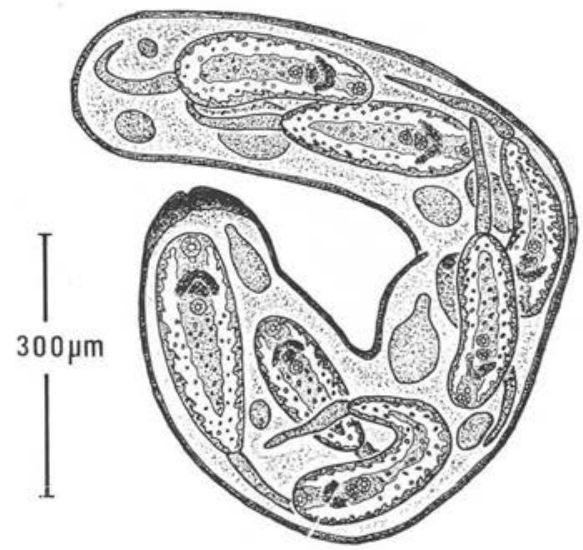

Fig. 1. Renicola lari, Cerithium mediterraneum. Sporocyste.

\section{Cercaire}

Les premières émergences de cercaires ont été observées dans la deuxième moitié du mois de juin; les dernières ont lieu vers la fin du mois de septembre ou au début du mois d'octobre. Les émissions, massives et discontinues, sont séparées par des intervalles de temps très variables; il semble que la température joue un rôle prépondérant dans ce phénomène.

A. - Description. Les dimensions ci-après sont celles de quinze cercaires émises spontanément par le Mollusque hôte.

Longueur du corps : 210-280 (240),

Largeur du corps : 104-134 (115).

Epaisseur du tégument : 2,5 .

Longueur des épines tégumentaires : 5.

Ventouse orale (vo): 18,5-26 (22) $\times$ 20-28 (23).

Ventouse ventrale $(\mathrm{vv}): 19,5-27(23,5) \times 21,5-28(24)$.

Rapport ventousaire vo/vv : $0,85-1,10(0,95)$.

Longueur précétabulaire (L) : 82-104 (90).

Longueur postacétabulaire (1) : 110-165 (127).

Rapport L/1 : 0,83-0,99 (0,95).

Primordium génital : 13,5-16 (15) × 24-28 (26).

Queue : $190-290(230) \times 18-26(21)$. 
Le tégument est entièrement spinulé (fig. 2). Les épines, disposées en quinconce, sont très peu distinctes.

Les ventouses ont une forme circulaire; elles sont subégales. Les dimensions de la ventouse orale sont cependant un peu inférieures à celles de l'acétabulum. La ventouse ventrale est disposée légèrement en avant du milieu du corps de la cercaire.

Les glandes céphaliques se répartissent en deux masses symétriques dans la région antéro-latérale de la ventouse ventrale (fig. 3). Les cellules, dont le nombre exact n'a pu être évalué, sont entourées par des granulations de couleur jaune-orangée. Des glandes céphaliques se détache un ensemble médian de canalicules sécréteurs s'ouvrant sur la lèvre dorsale de la ventouse orale, au niveau d'une dizaine de papilles disposées en deux grappes très proches et symétriques par rapport au plan sagittal. De part et d'autre de ce faisceau médian, un certain nombre de canalicules latéraux s'ouvrent à l'extérieur par une série de pores disposés de chaque côté, sur le bord latéral de la ventouse orale. L'ensemble des orifices des glandes céphaliques se place ainsi sur un demi-cercle correspondant à la bordure de la ventouse buccale.

Les glandes cystogènes sont peu nombreuses et de grande taille ; la plupart sont disposées dans la région post-acétabulaire.

De l'appareil digestif, seul est visible, avec difficulté, le pharynx globuleux, de 7 à $10 \mu \mathrm{m}$ de diamètre.

Un primordium génital s'observe en avant du bord antérieur de la ventouse ventrale. Il est limité latéralement par la bordure interne des glandes céphaliques.

L'appareil excréteur est du type mésostome (fig. 4). De chaque côté, les dix-huit protonéphridies se répartissent en deux ensembles, l'un préacétabulaire, l'autre postacétabulaire ; groupées trois par trois, leur disposition répond à la formule : $2[(3+3+$ $3)+(3+3+3)]=36$. Deux canaux excréteurs secondaires l'un antérieur, l'autre postérieur, s'unissent de chaque côté en un court canal principal se déversant dans la vessie à la hauteur de la ventouse ventrale. La vessie, très développée, est constituée d'une partie postérieure très courte et de deux bras remontant antérieurement jusque sur les côtés de la ventouse orale. Sur les côtés, la paroi vésicale présente une quinzaine de diverticules latéraux bien accusés; vers le plan médio-longitudinal, elle est seulement festonnée. Des granules réfringeants de petite taille sont visibles dans la lumière de la vessie; ils sont peu nombreux. Le pore excréteur s'ouvre à l'extrémité postérieure du corps.

Queue: La queue, simple, s'insère au fond d'une dépression postéroventrale du corps. Sa longueur est sensiblement égale à celle du corps de la larve. L'extrémité de la queue est légèrement granuleuse.

B. - Discussion. La systématique des cercaires de Renicolidae est actuellement très confuse. On a attribué au genre Renicola des cercaires de morphologies très différentes : les unes appartiennent au type Rhodométope, les autres au type Plagiorchide.

1. Les Cercaires Rhodométopes. Elles proviennent toutes de Turritella communis. La plupart ont été découvertes dans la Manche : Cercaria rhodometopa Pérez, 1924; 


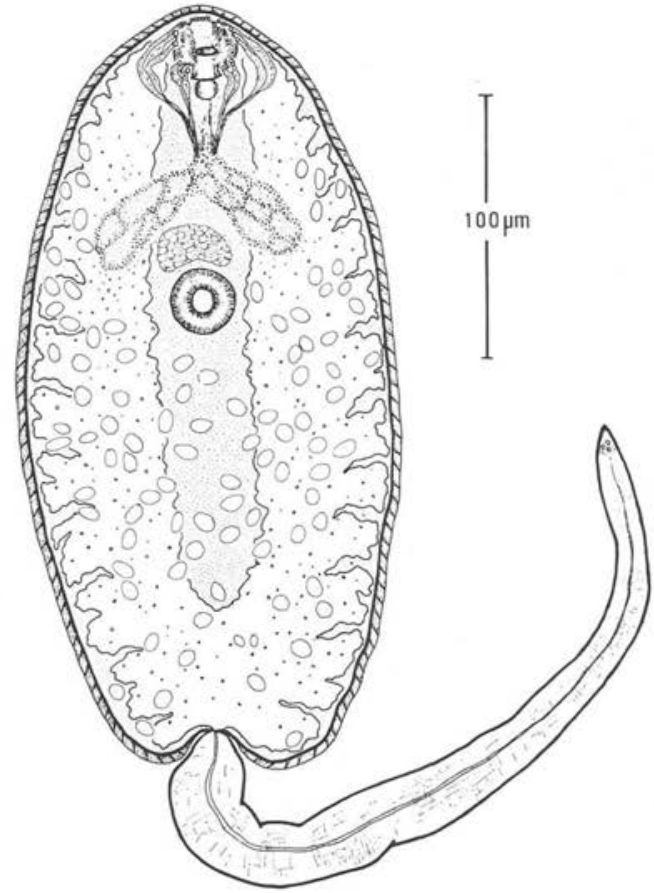

Fig. 2. Renicola lari. Cercaire. Morphologie générale (vue ventrale).

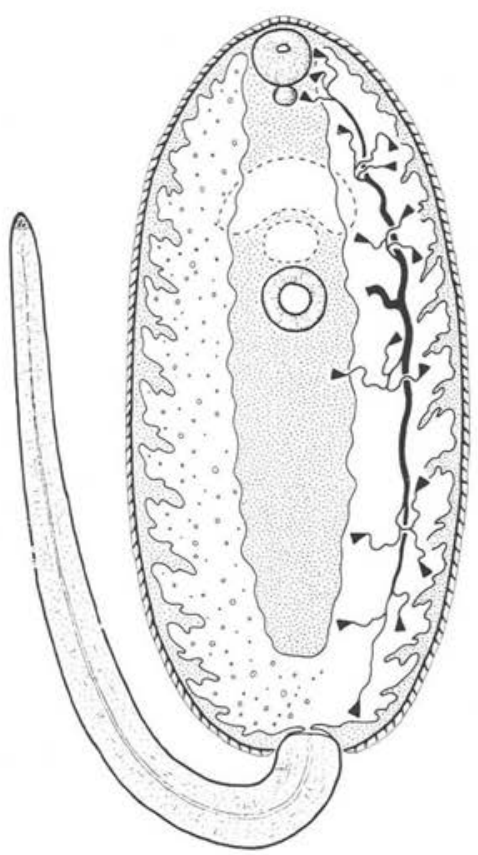

Fig. 4. Renicola lari. Cercaire. Système excréteur.

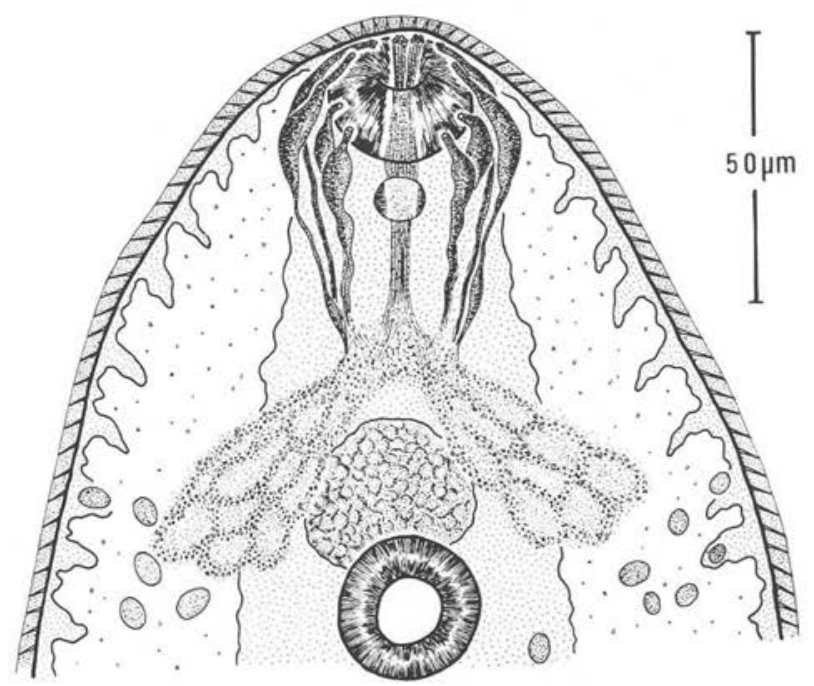

Fig. 3. Renicola lari. Cercaire. Région antérieure (vue ventrale). 
C. pythionike, C. herpsyllis, C. doricha, C. nicarete: M. Rothschild, 1935. D'autres sont connues de la Mer d'Irlande : C. doricha-pigmentata, C. cooki : C.A. Wright, 1956. D'autres enfin ont été décrites de Méditerranée : $C$. ampelis, $C$. ranzii : M. Rothschild, 1935.

Toutes ces cercaires se caractérisent par une queue bien développée pourvue de membranes ondulantes, par une vessie excrétrice en V, très volumineuse, s'étendant jusqu'à l'avant du corps et présentant des diverticules latéraux, par un système excréteur complexe paraissant toujours répondre à la formule :

$2[(n+n+n+n+n+n)+(n+n+n+n+n+n)]$ où $n=5$ ou 6.

2. Les cercaires du type Plagiorchide. Elles proviennent généralement de Mollusque Littorinidés ou Muricidés des rivages marins de l'ancien et du nouveau monde. Ce sont: Cercaria emasculans (P. Pelseneer, 1906) B.L. James, 1969 de Littorina littorea ; C. brevicauda (P. Pelseneer, 1906) B.L. James, 1969 de Littorina saxatilis tenebrosa; la cercaire de Renicola thaidus H.W. Stunkard, 1964 de Thais lapillus; la cercaire de $R$. roscovita (H.W. Stunkard, 1932) B. Werding, 1969 de Littorina littorea; Cercaria opaca R. B. Holliman, 1961 de Littorina irrorata ; C. littorinae-saxatilis VI A. Sannia et B. L. James, 1977 de Littorina saxatilis tenebrosa.

Ces cercaires sont caractérisées par un stylet, une petite queue dépourvue de voiles, une vessie excrétrice de dimensions réduites ayant la forme d'un $\mathrm{Y}$; leur formule excrétrice est du type $2[(3+3+3)+(3+3+3)]$, sauf chez $C$. brevicauda où elle est plus simple : $2[(3+3)+(3+3)]$. C. carribea XXXII et $C$. carribea XXCIII R. M. est plus simple : $2[(3+3)+(3+3)]$.C. carribea XXCII et $C$. carribea XXXIII R. M. Cable, 1956 proviennent de Cerithidea costata, la seconde se développe aussi chez Batillaria minima. C. queenslandae III L. R. G. Cannon, 1978 est issue de Cerithium moniliferum.

3. Entre ces deux groupes bien individualisés, se place un nombre important de cercaires de Renicolidae ne possédant pas la totalité des caractères de l'un ou de l'autre groupe; quelques autres cercaires, de type intermédiaire, présentent même un mélange des caractères Rhodométopes et Plagiorchides.

a) On peut rapprocher des cercaires Rhodométopes, celles dont la queue est bien développée et qui ont une vessie excrétrice dont les bras, pourvus d'expansions latérales, atteignent l'extrémité antérieure du corps. Ce sont : Cercaria carribea VII et $C$. carribea IX: R. M. Cable, 1956 hébergées respectivement par Tegula fasciata et par Cerithium variabilis; Cercaria carribea LXVIII R. M. Cable, 1963 parasite de Cerithium algicola.

b) Les cercaires qui se rapprochent du groupe Plagiorchide sont : Cercaria buchanani W. E. Martin et V.L. Grégory, 1951 de Cerithium californica; $C$. ingentis et C. nubeculata R. B. Holliman, 1961 de Cerithidea scalariformis; Renicola cerithidicola W. E. Martin, 1971 de Cerithidea californica. Ces cercaires sont toutes dépourvues de stylet mais leur queue est réduite et leur vessie excrétrice, de petite taille, a la forme d'un Y ; Cercaria ingentis, C. buchanani et la métacercaire de Renicola cerithidicola ont une formule excrétrice du type : $2[(3+3+3)+(3+3+3)]$. 
c) Deux cercaires présentent un mélange des caractères Plagiorchides et Rhodométopes. Ce sont : Cercaria carribea VIII R. M. Cable, 1956 de Cerithium algicola et la cercaire de Renicola lari ici décrite. Ces deux cercaires ont la formule excrétrice de type Plagiorchide : $2[(3+3+3)+(3+3+3)]$. Par contre, le grand développement et la forme de la vessie excrétrice les rapprochent du groupe Rhodometope. Il est difficile d'attribuer la cercaire de Renicola lari à l'un ou à l'autre de ces deux types; nous pensons cependant la rattacher au groupe Rhodométope en raison de son comportement planctonique.

C. - Comportement. La longévité de la cercaire est importante; nous l'avons estimée à 60 heures environ à $18^{\circ} \mathrm{C}$. Cependant, son activité ne dépasse pas 48 heures.

La nage de la cercaire, caractéristique, est comparable à celle observée chez certaines cercaires du type Rhodométope (M. Rothschild, 1935 ; C. A. Wright, 1956). Dès qu'elle sort du Mollusque, la large nage vers la surface (fig. $5 \mathrm{~A}$ ) ; quand elle y est arrivée, elle cesse ses mouvements et tombe ainsi vers le fonds, par gravité, pendant 5 à 10 secondes (fig. $5 \mathrm{~B}$ ) ; elle reprend alors son activité et remonte vers la surface pendant 30 à 60 secondes. Comme le signale C. A. Wright (1956), ces mouvements verticaux de montée et de descente caractérisent de nombreux animaux planctoniques, en particulier certains Copépodes qui constituent une grande partie de la nourriture des Poissons planctonophages.

L'attitude de la cercaire lors de la redescente est particulière : le corps est légèrement incurvé sur la face ventrale, cette dernière étant tournée vers le haut; la queue, dans le prolongement de la courbure du corps, est enroulée sur elle-même. Pendant la nage (mouvement ascendant), le corps de la cercaire a la forme d'un C ouvert latéralement; la queue, fonctionnant comme propulseur, l'amène vers la surface par de vigoureux battements latéraux.

Fig. 5. Renicola lari. Attitudes de la cercaire. A : au cours de son déplacement vers la surface (nage). B : au cours de sa descente vers le fond. Sur la gauche: vues latérales; sur la droite: vues par le haut.

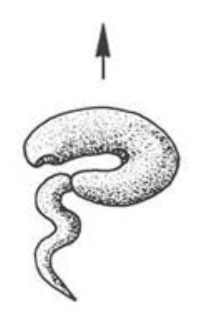

(A)
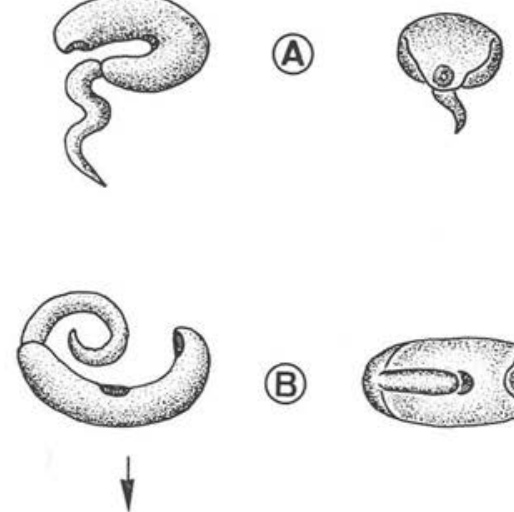

(B)

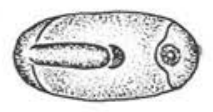




\section{Métacercaire}

\section{A. - Hôtes.}

- Les métacercaires de Rénicolidés ont été trouvées chez des Mollusques lorsquelles correspondent à des cercaires pourvues d'un stylet (type Plagiorchide). Ainsi, les cercaires de Renicola thaidus s'enkystent chez Mytilus edulis et chez Pecten irradians (H. W. Stunkard, 1964), celles de R. Roscovita chez Littorina littorea et Mytilus edulis (B. Werding, 1969) ; Cercaria opaca s'enkyste chez Littorina irrorata (R. B. Holliman, 1961) ; Cercaria brevicauda s'enkyste chez Littorina saxatilis tenebrosa (B. L. James, 1969). Exceptionnellement, Cercaria emasculans évolue en métacercaire chez Carcinus maenas et Cancer pagurus (B. L. James, 1969). A. Sannia et B. L. James (1977) décrivent une métacercaire progénétique parasitant Thais (Nucella) lapillus: Metacercaria nucellae-lapillus.

- Les métacercaires correspondant aux cercaires Rhodométopes sont localisées par contre dans le mésentère ou les caecums pyloriques de Poissons Clupéiformes (cas de Cercaria pythionike, C. doricha, C. rhodometopa) ou Gadiformes (cas de Cercaria doricha).

- Les métacercaires issues de larves intermédiaires entre les types Rhodométope et Plagiorchide, ont été trouvées aussi chez des Poissons. Cercaria buchanani s'enkyste dans le foie de Fundulus parvipinnis parvipinnis et de Gillichthys mirabilis (Téléostéens Cyprinodontiformes); Cercaria carribea IX évolue en métacercaire dans le foie de divers Gobiidae; Renicola cerithidicola s'enkyste dans les branchies de Fundulus parvipinnis parvipinnis.

- La métacercaire de Renicola lari se localise dans le foie de Atherina hepsetus L. et $A$. boyeri Risso: Téléostéens Mugiliformes.

B. - Kyste.

Diamètre : 225-345 (270).

Epaisseur de la paroi : 16-57 (34).

Diamètre de la cavité : 192-244 (225).

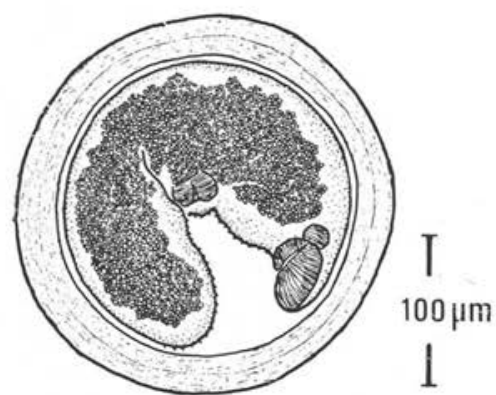

Fig. 6. Renicola lari. Métacercaire dans son kyste. Foie de Atherina boyeri.

Les kystes se détachent aisément des tissus hépatiques. Chacun d'eux renferme une métacercaire repliée sur elle-même, ses extrémités ne se touchant pas (fig. 6). La larve est animée de mouvements continuels, facilités par l'ampleur de la cavité kystique. Le diamètre de cette dernière varie dans des proportions peu importantes et la diversité de taille des kystes doit être attribuée essentiellement à une augmentation d'épaisseur de la paroi. La paroi est finement stratifiée; elle n'est pas attaquée par la trypsine que nous avons utilisée pour tenter le dékystement artificiel. 


\section{C. - Métacercaire.}

Longueur du corps: 210-352.

Largeur du corps : 110-160.

Ventouse orale (vo) : $24-42 \times 26-44$.

Ventouse ventrale (vv) : $24-40 \times 28-45$.
Rapport ventousaire (vo/vv) : 0,94-1,12.

Longueur préacétabulaire (L) : 116-178.

Longueur postacétabulaire (1) : 108-162.

Rapport L/1 : 0,96-1,10.

La comparaison de ces mensurations avec celles de la cercaire indique une croissance allométrique à l'intérieur du kyste. La partie préacétabulaire s'accroît plus rapidement que la région postacétabulaire; la ventouse orale grandit plus vite que la ventouse ventrale.

La morphologie de la métacercaire est en tous points comparable à celle d'une cercaire dépourvue de queue. Les épines tégumentaires sont cependant plus distinctes et le parenchyme plus transparent. La vessie excrétrice, énorme, a une forme identique à celle de la cercaire. Elle est emplie d'un très grand nombre de granulations sphériques homogènes (de $3 \mu \mathrm{m}$ à $4 \mu \mathrm{m}$ ), de couleur noirâtre qui donnent à la métacercaire une teinte générale très foncée.

\section{D. - Expérimentation.}

Le comportement particulier de la cercaire nous a conduits à penser que, comme l'avait déjà supposé C. A. Wright (1956), elle devait faire partie intégrante du plancton marin et, en tant que tel, pouvait être avalée par un Poisson.

Plusieurs expériences consistant à mettre dans un aquarium diverses espèces de Poissons de petite taille (Atherina boyeri, Mugil auratus, Syngnathus abaster, Gobius sp.) en présence de cercaires de Renicola lari, nous ont permis de constater que tous ces Poissons pouvaient se nourrir de cercaires. En effet, les larves ont été retrouvées vivantes et encore pourvues de leur queue dans l'intestin de ces divers Poissons.

Chez Gobius sp. et Syngnathus abaster, les cercaires survivent un certain temps dans le tube digestif et finissent vraisemblablement par être digérées. Chez Mugil auratus et Atherina boyeri, les cercaires passent par effraction à travers la paroi intestinale, abandonnant leur queue dans la lumière du tube digestif. Chez $M$. auratus, les cercaires dégénèrent, probablement dans la cavité générale. L'enkystement n'a lieu que dans le foie d'Atherina boyeri.

\section{E. - Contaminations naturelles.}

Le nombre de kystes hébergés par l'hôte varie avec la taille de ce dernier et selon l'époque de l'année. Une rapide estimation du nombre de métacercaires hépatiques de Renicola lari chez Atherina boyeri nous a permis d'évaluer approximativement leur abondance en fonction de la taille et de la saison de la capture du Poisson-hôte (tableau I).

L'émission des cercaires ayant lieu pendant l'été, les Athérines nées après cette période ne sont donc pas contaminées au mois de mai suivant. Par contre, la plupart de ces jeunes Poissons seront parasités en septembre. Les individus de taille moyenne ou grande ont été infestés dès l'année précédente. La durée de vie de la métacercaire peut être estimée à une année, probablement pas davantage, car des kystes en voie de dégé- 
Tableau I. Atherina boyeri - Renicola lari : variation de l'abondance des métacercaires en fonction de la taille de l'hôte et de la saison. Le Brusc (Var, France).

\begin{tabular}{|c|c|c|c|c|}
\hline \multicolumn{2}{|c|}{ Taille des Athérines (en mm) } & Jusqu’à 39 & $40-59$ & $60-85$ \\
\cline { 1 - 2 } $\begin{array}{c}\text { Abondance } \\
\text { des métacercaires }\end{array}$ & Mai ......... & 0,2 & 3,6 & 12,8 \\
\cline { 2 - 3 } & Septembre .... & 1,8 & 6 & 11 \\
\hline
\end{tabular}

nérescence se rencontrent assez fréquemment chez les Poissons de taille moyenne ou grande. Le pourcentage d'infestation des grands spécimens reste stable entre mai et septembre ; cette particularité laisse supposer qu'à partir d'une certaine taille ou d'un certain âge, le régime alimentaire des Athérines change. On peut donc penser qu'au delà d'une certaine taille, les cercaires n'entrent qu'exceptionnellement dans la chaîne alimentaire d'Atherina boyeri et $A$. hepsetus..

\section{Adulte}

\section{A. - Hôtes.}

En Provence, les hôtes définitifs naturels sont:

- Larus argentatus michaellis Naumann ; Goéland argenté à pieds jaunes.

- Larus ridibundus L.; Mouette rieuse.

\section{B. - Expérimentation.}

Des foies d'Atherina boyeri contaminées naturellement ont été donnés en nourriture à un Goéland et à une Mouette élevés en laboratoire, donc indemnes de tout parasite. Chaque Oiseau a été contaminé à deux reprises, à dix jours d'intervalle. Le Goéland a été sacrifié 55 jours après le premier repas infestant, la Mouette 60 jours après la première infestation. Nous avons obtenu 330 Renicola lari adultes chez le Goéland, 74 chez la Mouette.

\section{C. - Habitat.}

Renicola lari se rencontre exclusivement dans les canaux urinifères des reins; cette localisation a été décrite de façon détaillée par J. Timon-David (1933). Nous avons constaté que la distribution des parasites dans les reins des hôtes définitifs est loin d'être homogène. Les Renicola lari, logés par couples dans les cônes médullaires, sont d'autant plus abondants que l'on est plus près de l'extrémité postérieure des reins, donc du point de départ des uretères; ils se raréfient au fur et à mesure que l'on se rapproche des lobes rénaux antérieurs (tableau II).

Même en tenant compte du fait que le lobe postérieur est le plus développé, une telle distribution implique incontestablement que la migration de la métacercaire infestante se fait par voie urinaire. Les métacercaires sont entraînées jusqu'au cloaque d'où elles remontent activement le long des uretères pour venir se loger dans leur habitat 
Tableau II. Variation du nombre de Renicola lari adultes aux différents niveaux du rein droit d'un Goéland et d'une Mouette parasités expérimentalement.

\begin{tabular}{|c|c|c|c|c|}
\hline \multicolumn{2}{|c|}{ Rein droit } & Lobe antérieur & Lobe moyen & Lobe postérieur \\
\hline \multirow{2}{*}{$\begin{array}{l}\text { Nombre de } \\
\text { Renicola lari }\end{array}$} & Goéland.... & 16 & 10 & 140 \\
\hline & Mouette $\ldots \ldots$ & 2 & 8 & 24 \\
\hline
\end{tabular}

d'élection. La découverte d'une post-larve encore dans l'uretère d'un Goéland naturellement contaminé confirme cette opinion.

\section{D. - Dimensions.}

Le nombre important d'individus adultes obtenus après infestation expérimentale, nous permet de donner de façon précise les mensurations de ce parasite. Nous soulignons que l'étude anatomique du ver adulte est rendue difficile en raison du grand développement de l'utérus qui, bourré d'œufs, cache la majorité des organes (fig. 7).

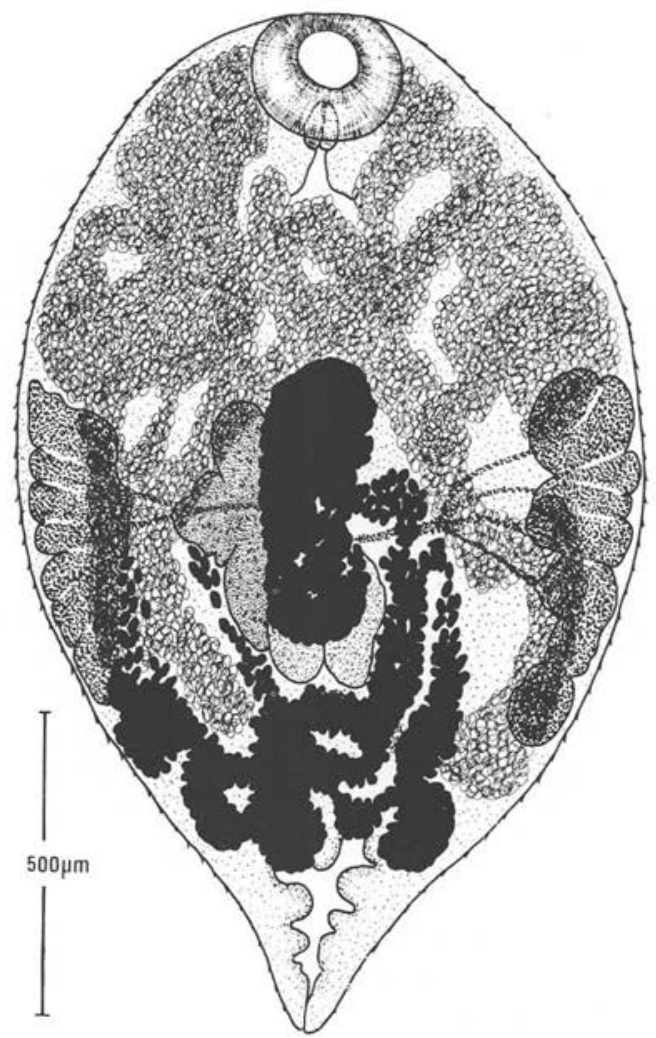

Fig. 7. Renicola lari, Larus argentatus. Adulte expérimental renfermant beaucoup d'œufs (vue ventrale). 
Les dimensions sont celles de 16 individus provenant du Goéland argenté (symbolisées par la lettre G) et de 20 spécimens récoltés chez la Mouette rieuse (symbolisées par la lettre M).

Longueur du corps :

G : $1225-1945$ (1 448) ;

M : $1225-1895$ (1 518).

Largeur du corps :

G : 560-1 039 (823) ;

M : 682-1 311 (894).

Longueur préacétabulaire (L) :

G : 709-1 092 (884) ;

M: 746-1 180 (896).

Longueur postacétabulaire (1) :

G : 293-773 (481) ;

M : 346-719 (539).

Rapport L/1 :

$\mathrm{G}: 1,34-2,66(1,91)$;

M : $1,33-3,32(1,70)$.

Ventouse orale (vo):

$\mathrm{G}: 158-227(193) \times 195-270(232)$;

M : 168-254 (202) $\times 195-284$ (238).

Ventouse ventrale (vv) :

G: $76-101(85) \times 72-102(87)$;

M : 72-106 (85) × 72-109 (88).

Rapport ventousaire vo/vv :

$\mathrm{G}:$ : 2,02-2,64 $(2,28)$;

M : $2,02-3,00(2,40)$.
Pharynx (P) :

$\mathrm{G}: 65-86(76) \times 54-80(65)$;

$\mathrm{M}: 65-89(74) \times 56-73(63)$.

Rapport vo/P :

$\mathrm{G}: 2,32-3,00(2,56)$;

M : $2,17-3,50(2,77)$.

Ovaire :

$\mathrm{G}: 256-435(357) \times 80-238(146)$;

$M: 220-540(406) \times 100-300(143)$.

Testicule droit :

G : $100-217(163) \times 48-135$ (177) ;

M: $152-280(206) \times 85-108(92)$.

Testicule gauche:

G: $130-220(165) \times 32-117(75)$;

Sac utérin :

M : $150-240(194) \times 70-108(91)$.

CEufs:

G: $100-320(203) \times 54-260(152)$;

$M: 120-330(212) \times 100-195(130)$.

G : $28-36(33,3) \times 15-21(18,0)$;

M : $29-35(32,0) \times 15-21(17,5)$.

Vitellogène droit :

G : $205-540$ (358) × 86-200 (138) ;

$M: 270-560$ (413) $\times 86-220$ (143).

Vitellogène gauche :

G: $217-590(391) \times 76-217$ (141) ;

$M: 345-580(445) \times 87-240$ (151).

\section{E. - Précisions complémentaires sur l'anatomie.}

Les dimensions et les particularités anatomiques de nos spécimens sont conformes à celles données par J. Timon-David (1933). Cependant quelques points concernant les appareils génitaux et excréteur doivent être précisés.

1. Appareil génital mâle (fig. 8). Très fréquemment, les testicules se chevauchent dans leur portion distale et nous avons pu constater, chez un exemplaire vivant, une fusion des deux parties recouvrantes. Les canaux déférents confluent au ras de l'extrémité postérieure de la vésicule séminale. Le canal éjaculateur est court, non musculeux ; il n'est pas exsertile mais est sans cesse animé de petits frémissements : il n'y a donc pas de cirre. Les cellules prostatiques constituent un manchon enveloppant la vésicule séminale et le canal éjaculateur. Il n'y a pas de poche du cirre.

2. Appareil génital femelle (fig. 8 et 9). L'oviducte se détache de la portion médiane et interne de l'ovaire ; il est très court. Au-delà du réceptacle séminal se trouve la chambre de fécondation; sa ciliature refoule les spermatozoïdes en amont. Le vitelloducte impair est assez allongé ; il débouche dans l'utérus immédiatement en avant de la glande 


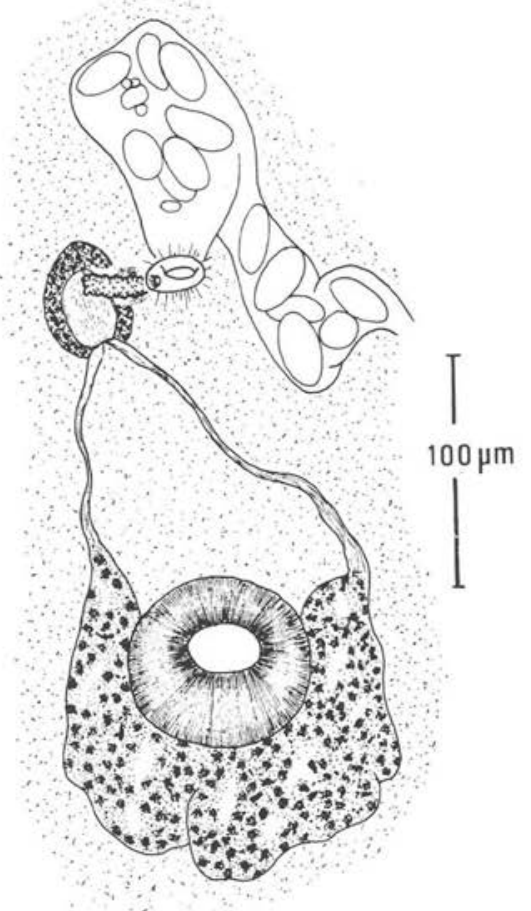

Fig. 8.
Fig. 8. Renicola lari, Larus argentatus. Adulte expérimental. Appareil génital mâle et extrémité distale de l'appareil génital femelle (vue ventrale).

Fig. 9. Renicola lari, Larus argentatus. Adulte expérimental. Région proximale de l'appareil génital femelle (vue ventrale).

Fig. 10. - Renicola lari, Larus argentatus. Adulte expérimental renfermant un petit nombre d'œufs (vue ventrale).

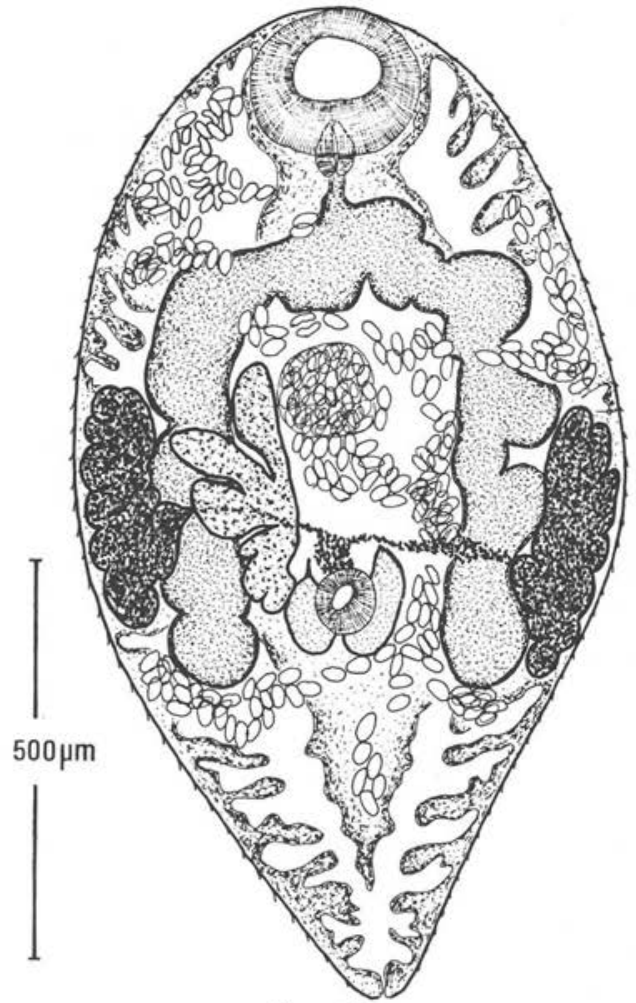

Fig. 10.

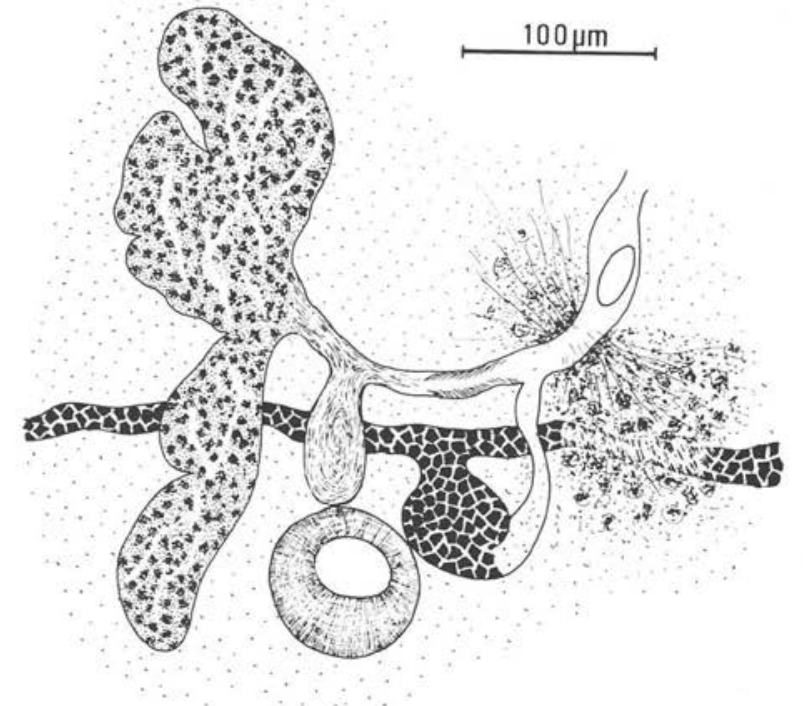

Fig. 9. 
de Mehlis. L'utérus décrit de nombreuses circonvolutions. A son extrémité distale il constitue une poche de taille variable, le sac utérin. Celui-ci est disposé en avant du pore génital, dans la région médiane. Chez les jeunes individus, le sac utérin est relativement petit ; chez les exemplaires âgés de grande taille, ce sac est bourré d'un nombre corisidérable d'œufs à paroi très sombre, prêts à être pondus. Le sac utérin est en relation avec un petit atrium génital s'ouvrant à l'extérieur par le pore génital médian.

3. Appareil excréteur. Comme chez la cercaire et chez la métacercaire, la vessie excrétrice de l'adulte débute par deux branches naissant de part et d'autre de la ventouse orale et se réunissant en un court tronc médian et postérieur (fig. 10). Dans chaque branche, la paroi latérale située en bordure du corps est largement digitée ; celle située en regard du plan sagittal est seulement festonnée. Il ne nous a pas été possible de vérifier si la formule excrétrice du ver adulte était identique à celle de la cercaire.

\section{F. - Discussion.}

La croissance de Renicola lari est en tous points identique chez le Goéland et chez la Mouette ; en effet, les mensurations des individus obtenus expérimentalement chez ces deux Oiseaux se superposent parfaitement.

Les dimensions des spécimens naturels décrits par J. Timon-David (1933) correspondent à celles que nous avons données. Il y a cependant deux exceptions : la taille de l'ovaire et celle des œufs.

En ce qui concerne l'ovaire, il semble que J. Timon-David ait donné des dimensions se rapportant à de petits individus car l'ovaire n'est bien distinct que chez ces exemplaires là $(200-220 \mu \mathrm{m} \times 80-130 \mu \mathrm{m})$. Chez les vers de grande taille, les dimensions de l'ovaire sont difficiles à mesurer en raison du grand développement de l'utérus.

Les dimensions des œufs indiquées par J. Timon-David $(48-50 \mu \mathrm{m} \times 27-28 \mu \mathrm{m})$ sont par contre très nettement supérieures à celles que nous avons données (28-36 $\mu \mathrm{m} \times$ 15-21 $\mu \mathrm{m}$ ). Une telle différence est d'autant plus surprenante que J. Timon-David souligne la faible variation des dimensions des œufs. C'est en effet ce que nous avons constaté chez chaque individu pris séparément. Par contre, la comparaison des mensurations d'œufs provenant d'exemplaires différents indique une grande hétérogénéité et cela en dépit de l'âge identique des individus obtenus par développement expérimental. Le degré de développement de la métacercaire au moment de la contamination ne peut rendrc compte de ce phénomène. En effet, les œufs de grande taille peuvent être observés aussi bien chez des parasites relativement peu développés que chez des individus beaucoup plus évolués; il ne peut donc être question ici que de variations morphologiques intraspécifiques.

Nous attribuons à Renicola lari les individus que nous avons obtenus expérimentament dans les reins de Larus argentatus et $L$. ridibundus. Nous rappelons que $R$. lari a déjà été signalé chez de nombreux Lariformes des genres Hydrochelidon, Sterna, Hydroprogne, Chlidonias en Europe et en Sibérie. 
La systématique des Renicolidae adultes reste actuellement encore très confuse en raison du peu d'individus généralement utilisés pour les descriptions des espèces, de la difficulté des observations microscopiques et des variations morphologiques individuelles auxquelles s'ajoute une grande similitude interspécifique. Dans ces conditions, il apparaît que seule la connaissance des cycles biologiques peut permettre une mise en ordre du genre Renicola, genre hétérogène au niveau des cercaires et donnant au contraire une fausse impression d'homogénéité au niveau des adultes.

\section{Bibliographie}

Cable R.M., 1956: Marine cercariae of Puerto Rico. Sci. Survey Porto-Rico and Virgin Islands. N.Y. Acad. Sci., 16, 491-577.

Cable R.M., 1963 : Marine cercariae from Curaçao and Jamaïca. Z. Parasitenk., 23, 429-469.

Cannon L.R.G., 1978: Marine cercariae from the gastropod Cerithium moniliferum Kiener at Heron Island, Great Barrier Reef. Proc. R. Soc. Qd, 89, 45-57.

Dollfus R.Ph., 1946: Sur un distome du genre Tamerlania K.I. Skrjabin, 1924, avec un catalogue des Trématodes des reins d'Oiseaux. Ann. Parasitol. Hum. Comp., 21, 25-73.

Holliman R.B., 1961: Larval trematodes from the Apalachee Bay area, Florida, with a checklist of known marine cercariae arranged in a key to their superfamilies. Tulane Stud. Zool., 9, $2-74$.

James B.L., 1969 : The Digenea of the intertidal Prosobranch, Littorina saxatilis (Olivi). Sonderd. Z. f. Zool. Systematik Evolutionsforschung, 7, 273-316.

Mackenzie K., 1975: Renicola metacercariae (Digenea : Renicolidae) in Clupeoid fish : new host records. J. Fish. Biol., 7, 359-360.

Martin W.E., 1971: Larval stages of Renicolid Trematodes. Trans. Am. Microsc. Soc., 90, 188-194.

Martin W.E., Gregory V.L., 1951: Cercaria buchanani n. sp., an aggregating marine trematode. Trans. Am. Microsc. Soc., 60, 359-362.

Rothschild M., 1935: The Trematode parasites of Turritella communis Lmk from Plymouth and Naples. Parasitol., 27, 152-170.

Sannia A., James B.L., 1977: The digenea in marine molluscs from Eyjafjordur, north Iceland. Ophelia, 16, 97-109.

Stunkard H.W., 1932: Some larval Trematodes from the coast in the region of Roscoff, Finistère. Parasitol., 24, 321-343.

Stunkard H.W., 1964 : Studies on the Trematode genus Renicola : observations on the life-history, specificity, and systematic position. Biol. Bull., 126, 467-489.

Stunkard H.W., 1971: Renicolid Trematodes (Digenea) from the renal tubules of Birds. Ann. Parasilol. Hum. Comp., 46, 109-118.

Timon-David J., 1933: Sur une nouvelle espèce de Renicola, Trématode parasite du rein des Laridés. Bull. Inst. Oceanogr. Monaco, 616, 1-16.

Timon-David J., 1953 : Sur une métacercaire de la Sardine et ses affinités avec le groupe Rhodometopa. C.R. Acad. Sci. Paris, 237, 1182-1184.

Timon-David J., 1955: Trématodes des Goélands de l'île de Riou. Ann. Parasitol. Hum. Comp., 30, 446-476.

Werding B., 1969: Morphologie, Entwicklung und Okologie digener Trematoden-Larven der Strandschnecke Littorina littorea. Marine Biol., 3, 306-333.

Wright C.A., 1956: Studies on the life history and ecology of the Trematode genus Renicola Cohn, 1904. Proc. Zool. Soc. London, 126, 1-49. 